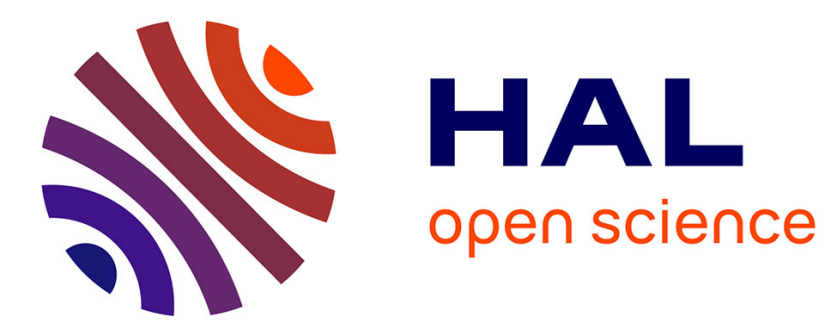

\title{
Non-local versus local behaviour of microheterogeneous metals
}

\author{
M. Berveiller
}

\section{To cite this version:}

M. Berveiller. Non-local versus local behaviour of microheterogeneous metals. Journal de Physique IV Proceedings, 1993, 03 (C7), pp.C7-621-C7-628. 10.1051/jp4:19937100 . jpa-00251717

\section{HAL Id: jpa-00251717 https://hal.science/jpa-00251717}

Submitted on 1 Jan 1993

HAL is a multi-disciplinary open access archive for the deposit and dissemination of scientific research documents, whether they are published or not. The documents may come from teaching and research institutions in France or abroad, or from public or private research centers.
L'archive ouverte pluridisciplinaire HAL, est destinée au dépôt et à la diffusion de documents scientifiques de niveau recherche, publiés ou non, émanant des établissements d'enseignement et de recherche français ou étrangers, des laboratoires publics ou privés. 


\title{
Non-local versus local behaviour of microheterogeneous metals
}

\author{
M. BERVEILLER
}

Laboratoire de Physique et Mécanique des Matériaux, URA 1215 du CNRS, ENIM, Ile du Saulcy, 57045 Metz, France

\begin{abstract}
Modelization of the elastoplastic behavior of metallic polycrystals based on micro-macro transitions using the powerful self-consistent scheme was developed for a local constitutive equation at the single crystal level.

Numerical results concerning yield surface, subsequent yield surface, induced texture and plastic as well as elastic anisotropy, second order residual stresses and stored energy are in good agreement with experimental results.

Despite this success some basic aspects of plasticity are not taken into account in the usual self-consistent scheme with local behavior at the grain level. They concern mainly the process of heterogeneization by plastic straining related to the spontaneous formation of dislocation pattern which is described through non-local as well as convective effects.
\end{abstract}

\section{INTRODUCTION}

During large elastoplastic straining of polycrystals, a deep change of the material internal state is commonly observed, leading to a strong path-dependent overall behavior. Usual phenomenological descriptions of such a behavior need numerous parameters rather difficult to identify since the material is described in this case by a homogeneized medium.

On the other hand methods based on scale transition try to incorporate more microstructure and micromechanisms by describing the material as a continuous medium with microstructure and introducing different deformation mechanisms.

This review deals with the self-consistent scheme proposed by KRÖNER [1] and HILL [2] developed by BERVEILLER et ZAOUI [3, 4], LIPINSKI et BERVEILLER [5], which has been used to describe the behavior of polycrystalline materials in case of complex loading paths. Several hypothesis are used, like the crystallographic glide on well-defined planes and the associated Schmid law; in the same way, viscosity is neglected in case of room-temperature plastic straining.

In the polycrystalline case, several kinds of heterogeneity have to be considered:

- due to the misorientation of grains (i.e. the crystallographic texture), a heterogeneous and incompatible plastic strain field induced among grains leads to internal stresses of the second order,

- at the intragranular level, dislocation substructures are induced which correspond to the heterogeneization occuring during plastic flow. Such features can lead to a high level of internal stresses inside the grains introducing non-local and convective effects,

- as a consequence of this, microshear bands may develop inside the grains and finally become transgranular; this latter point will not be discussed here. 


\section{SCALE TRANSITION WITH UNIFORM GRAIN BEHAVIOR}

As a matter of fact, an efficient way of describing the behavior of polycrystalline materials considers the polycrystal as a piece-wise uniform medium at the level of grains, so that homogeneous crystallographic multislip is assumed in the grains. Then from the knowledge of the single crystal behavior the selfconsistent scheme allows to achieve the scale transition to the polycrystal level through localization and further homogeneization operations.

\subsection{Single crystal behavior}

For usual FCC or BCC crystal, the elastic behavior is linear and may be defined in case of isotropy by the Lamé constants $\lambda$ and $\mu$. FCC single crystals possess 12 easy glide systems $\{111\}<110>$ which may glide in the two senses. The situation for BCC materials seems more complicated since the pencil-glide which occurs there is described by two families $\{110\}<111>$ and $\{112\}<111>$.

The single crystal velocity gradient $\mathbf{g}$ is decomposed in the strain rate $\mathbf{d}$ and spin $\mathbf{w}$ :

$$
\mathbf{g}=\mathbf{d}+\mathbf{w}
$$

which in turn may be split into elastic and plastic parts

$$
g=d^{e}+d^{p}+w^{e}+w^{p}
$$

The plastic part of $\mathbf{g}$ results from the combination of the plastic slip rates $\dot{\gamma}^{8}$ on active glide systems $g$. Let $\overrightarrow{\mathbf{n}}^{g}$ be the normal vector to a glide plane and $\overrightarrow{\mathbf{m}}^{g}$ the slip direction in this plane.

The following usual projection relations are obtained :

$$
\begin{aligned}
& \mathrm{d}_{\mathrm{ij}}^{\mathrm{p}}=\frac{1}{2} \sum_{g}\left(\mathrm{~m}_{\mathrm{i}}^{g} n_{\mathrm{j}}^{g}+\mathrm{m}_{\mathrm{j}}^{g} \mathrm{n}_{\mathrm{i}}^{g}\right) \dot{\gamma}^{g}=\sum_{g} \mathrm{R}_{\mathrm{ij}}^{g} \dot{\gamma}^{g} \\
& \mathrm{w}_{\mathrm{ij}}^{\mathrm{p}}=\frac{1}{2} \sum_{g}\left(\mathrm{~m}_{\mathrm{i}}^{g} n_{\mathrm{j}}^{g}-\mathrm{m}_{\mathrm{j}}^{g} \mathrm{n}_{\mathrm{i}}^{g}\right) \dot{\gamma}^{g}=\sum_{g} \mathrm{Q}_{\mathrm{ij}}^{g} \dot{\gamma}^{g}
\end{aligned}
$$

On the other hand, the elastic constitutive relation for single crystal may be written as following, in the large strains formulation, :

$$
\stackrel{\nabla}{\sigma_{i j}}{ }^{*}=C_{i j k l} d_{k l}^{e}-\sigma_{i j} j_{k k}^{e}
$$

where ${ }^{\nabla}{ }_{i j}{ }^{*}$ is the rate of Cauchy stress with respect to the lattice and $C_{i j k l}$ are the elastic moduli.

In case of large strains, it is convenient to introduce the nominal stress tensor $\mathbf{n}$ in order to facilitate the averaging operations; we have :

$$
\dot{\mathrm{n}}_{\mathrm{ij}}=\dot{\sigma}_{\mathrm{ij}}-\left(\mathrm{d}_{\mathrm{ik}}+\mathrm{w}_{\mathrm{ik}}\right) \sigma_{\mathrm{kj}}+\sigma_{\mathrm{ij}} \mathrm{d}_{\mathrm{kk}}
$$

Then by applying the Schmid law to describe the plastic slip on an active system $g$, we have :

$$
\left\{\begin{array}{c}
\tau^{g}=\tau_{\mathrm{c}}^{g} \\
\dot{\tau}^{g}=\dot{\tau}_{\mathrm{c}}^{g}
\end{array}\right.
$$

where : $-\tau^{g}$ is the resolved shear stress on system $g$ and $\dot{\tau}^{g}=\sum_{i, j} R_{i j}^{g}{ }_{i j}^{\nabla}{ }^{*}$,

$-\tau_{\mathrm{c}}^{g}$ is the critical shear stress on system $g$ and $\dot{\tau}_{\mathrm{c}}^{g}=\sum_{h} \mathrm{H}^{g h} \dot{\gamma}^{h}$, 
$\mathrm{H}^{\mathrm{g} h}$ is the commonly used hardening matrix describing in an averaged maner the processes of hardening on system $g$ due to slip on system $h$.

The single crystal behavior is finally expressed by

$$
\dot{\mathrm{n}}_{\mathrm{ij}}=\mathrm{l}_{\mathrm{ijk1}} \mathrm{g}_{\mathrm{kl}}
$$

where I are the intanstaneous elastoplastic moduli of the grain.

Actually $l$ is strongly heterogeneous from grain to grain due to several facts :

- second order internal stresses,

- different orientations of the slip systems,

- critical shear stresses evolving differently,

so that a single phase polycrystal may be treated as a microheterogeneous medium.

\subsection{Scale transition}

Using Green functions, an integral equation is established which links the local velocity gradient of the inhomogeneous medium to the kinematic or static conditions imposed on the solid's external boundary:

$$
g_{i j}(r)=g_{i j}^{0}(r)+\int_{V} \Gamma_{i j k 1}^{0}\left(r-r^{\prime}\right) \delta l_{k l m n}\left(r^{\prime}\right) g_{m n}\left(r^{\prime}\right) d V^{\prime}
$$

where $\Gamma^{0}$ is the modified Green tensor and $\delta \mathbf{I}=\mathbf{I}-\mathbf{L}^{\theta}$ the heterogeneity of behavior between the grain and the macroscopic homogeneous reference medium with moduli $\mathrm{L}^{0}$.

By taking the symmetrical and antisymmetrical parts of (8), two equations are deduced which allow to get almost formally the strain rate and spin tensors :

$$
\begin{aligned}
& \dot{\varepsilon}_{\mathrm{ij}}(\mathrm{r})=\dot{\mathrm{E}}_{\mathrm{ij}}+\int_{\mathrm{V}} \Gamma_{\mathrm{ijkk}}^{0 \mathrm{~S}}\left(\mathrm{r}-\mathrm{r}^{\prime}\right) \delta \mathrm{I}_{\mathrm{klmn}}\left(\mathrm{r}^{\prime}\right) \dot{\varepsilon}_{\mathrm{mn}}\left(\mathrm{r}^{\prime}\right) \mathrm{d} \mathrm{V}^{\prime} \\
& \dot{\omega}_{\mathrm{ij}}(\mathrm{r})=\dot{\Omega}_{\mathrm{ij}}+\int_{\mathrm{V}} \Gamma_{\mathrm{ijkl}}^{0 \mathrm{AS}}\left(\mathrm{r}-\mathrm{r}^{\prime}\right) \delta \mathrm{I}_{\mathrm{klmn}}\left(\mathrm{r}^{\prime}\right) \dot{\varepsilon}_{\mathrm{mn}}\left(\mathrm{r}^{\prime}\right) \mathrm{d} \mathrm{V}^{\prime}
\end{aligned}
$$

where $\dot{\mathbf{E}}$ and $\dot{\Omega}$ are the imposed kinematic conditions at the boundary of the polycrystal.

To solve these localization equations, some assumptions and approximations are needed among which one finds :

- Taylor-Lin: $\quad \forall \mathrm{r}, \quad \dot{\varepsilon}(\mathrm{r})=\dot{\mathrm{E}}$

- Sachs: $\quad \forall \mathrm{r}, \quad \dot{\sigma}(\mathrm{r})=\dot{\Sigma}$

- Born approximation :

1st step

$$
\begin{aligned}
& \dot{\varepsilon}_{i j}^{1}(r)=\left(I_{i j m n}+\int_{V} \Gamma_{i j k l}^{0 S}\left(r-r^{\prime}\right) \delta l_{k l m n}\left(r^{\prime}\right) d V^{\prime}\right) \dot{E}_{m n} \\
& \dot{\varepsilon}_{i j}^{2}(r)=\dot{E}_{i j}+\int_{V} \Gamma_{i j k l}^{0 S}\left(r-r^{\prime}\right) \delta 1_{k l m n}\left(r^{\prime}\right) \dot{\varepsilon}_{i j}^{1}\left(r^{\prime}\right) d V^{\prime}
\end{aligned}
$$

2nd step

$$
=\left(I_{i j m n}+\int_{V} \Gamma_{i j \mathrm{jl}}^{0 S} \delta 1_{k l m n} d V^{\prime}+\int_{V} \Gamma_{i j k l}^{0 S} \delta 1_{k l p q} \Gamma_{p q r s}^{0 S} \delta 1_{\text {rsmn }} d V^{\prime}\right) \dot{E}_{m n}
$$

etc, ... 


\section{- Self-consistent scheme:}

Using the following decomposition of $\Gamma$ (KRÖNER [6]):

$$
\Gamma^{0 S}\left(r-r^{\prime}\right)=\Gamma^{1} \delta\left(r-r^{\prime}\right)+\Gamma^{2} \frac{1}{\left|r-r^{\prime}\right|^{3}} F(\varphi, \psi),
$$

equation (9) may be rewritten as

$$
\dot{\varepsilon}_{i j}(r)=\left(\dot{E}_{i j}+\Gamma_{i j m n}^{l} \delta l_{m n k l}(r) \dot{\varepsilon}_{k l}(r)+\int_{V} \Gamma_{i j k s}^{2} \frac{1}{\left|r-r^{\prime}\right|^{3}} \delta i_{k l m n}\left(r^{\prime}\right) \dot{\varepsilon}_{m n}\left(r^{\prime}\right) d V^{\prime}\right)
$$

By choosing the reference homogeneous medium $\mathrm{L}^{\circ}$ equal to the effective moduli $\mathrm{L}^{\text {eff }}$, the integral part in (11) becomes neglectible in comparison with the other terms. KRÖNER [6] showed that this solution is exact for the perfectly disordered media.

Then in case of uniform variables $\delta I^{I}, \dot{\varepsilon}^{I}$ inside the assumed ellipsoïdal grains, one obtains the localization tensor :

$$
\dot{\varepsilon}^{\mathrm{I}}=\left(\mathrm{I}-\Gamma^{\mathrm{I}} \Delta \mathrm{I}^{\mathrm{I}}\right)^{-1} \dot{\mathrm{E}}=\mathrm{A}^{\mathrm{I}} \dot{\mathrm{E}}
$$

From the knowledge of the single crystal behavior

$$
\dot{\sigma}^{\mathrm{I}}=1^{\mathrm{I}} \dot{\varepsilon}^{\mathrm{I}},
$$

the homogeneization operation is achieved to get the overall behavior

or

$$
\begin{gathered}
\dot{\mathbf{\Sigma}}=\left\langle\dot{\sigma}^{\mathrm{I}}\right\rangle=\left\langle\mathrm{I}^{\mathrm{I}} \dot{\varepsilon}^{\mathrm{I}}\right\rangle \\
=\left\langle\mathrm{I}^{\mathrm{I}} \dot{\boldsymbol{\varepsilon}}^{\mathrm{I}}\right\rangle \dot{\mathrm{E}} \\
\dot{\mathbf{\Sigma}}=\mathrm{L}^{\text {eff }} \dot{\mathrm{E}} \quad \text { where } \mathrm{L}^{\mathrm{eff}}=\left\langle\mathrm{I}^{\mathrm{I}} \mathrm{A}^{\mathrm{I}}\right\rangle
\end{gathered}
$$

Applications to FCC polycrystals were extendly presented in LIPINSKI et al. [7].

Many applications have shown interesting results, which have been successfully compared to experiments. These results concern texture evolution as well as second order internal stresses, stored energy, Lankford coefficient during complex loading paths (ex: cold rolling+ironing+necking) REUBREZ et al. [8], implementation in a Finite Element software SCACCIATELLA [9] or the problem of a polycrystal with intragranular precipitates SCHMITT et al. [10].

However this averaged description of the single crystal behavior does not take into account the progressive heterogeneization which occurs in the grains during plastic straining despite its basic importance especially at large strains. Thus it seems necessary to describe more accurately the single crystal behavior.

\section{DESCRIPTION OF THE INTRAGRANULAR BEHAVIOR}

A first attempt of representing the mechanical consequences of induced dislocation substructures, using micromechanical tools, was based on a formal non-local formulation particularized to the case of a twophase composite (BERVEILLER et al. [11]). The model was improved, introducing orientation and morphological anisotropy of the cells (MULLER et al. [12]).

However, this formulation ignores a basic feature of the cell structure organization, i.e. the reorientation of cells during complex loading paths. Thus it seems important to take into account the convective motion of dislocation networks, which should be immobile with respect to parts of the crystal lattice but mobile relatively to a fixed reference frame. 
This convective approach is developed and applied to the problem of a plastic inclusion. Through a twophase non-local description of the cell structure, the behavior of a grain with a dislocation cell structure is recovered.

\subsection{Non-local hardening}

A dislocation cell structure is composed of interiors, regions of low dislocation density, and walls with a higher dislocation density. This assumed polarized dislocation distribution, typical of low-temperature deformation, results from the displacement of mobile loops whose ends are embedded in the neighboring forest.

Since practically all dislocations produced during deformation (particularly through Frank and Read sources) move through the cell interiors toward the walls of the corresponding system where they are slowed down and finally stopped (and thus stored there), plastic glide inside the cell interiors increases the dislocation density and then the critical shear stress in the walls.

This is the so-called non-local hardening at the dislocation cell level: plastic glide at one place of a heterogeneous medium hardens another place of it.

This phenomenon is translated into micromechanics in order to describe the heterogeneization which occurs in the intragranular medium during plastic straining.

In classical local plasticity, a commonly used hardening matrix $\mathrm{H}$ is introduced in order to describe the hardening of slip systems, including the notion of latent hardening. This matrix links the critical shear stress rate $\dot{\tau}_{\mathrm{c}}^{g}(\mathrm{r})$ on slip system $g$ at point $\mathrm{r}$ with slip rates $\dot{\gamma}^{h}(\mathrm{r})$ on all active glide systems at the same point $r$ :

$$
\dot{\tau}_{\mathrm{c}}^{g}(\mathrm{r})=\sum_{h} \mathrm{H}^{g h}(\mathrm{r}) \dot{\gamma}^{h}(\mathrm{r})
$$

The dislocation interactions and storage processes described above cannot be described by such an averaged formulation so that one may introduce formally a non-local hardening matrix which is able to take them into account :

$$
\dot{\tau}_{\mathrm{c}}^{g}(\mathrm{r})=\int_{\mathrm{V}} \sum_{h} \mathrm{H}^{g h}\left(\mathrm{r}, \mathrm{r}^{\prime}\right) \dot{\gamma}^{h}\left(\mathrm{r}^{\prime}\right) \mathrm{dV} \mathrm{V}^{\prime}
$$

$\mathrm{H}$ allows spatial interactions from plastic slip rate on a system at point $\mathbf{r}^{\prime}$ with critical shear stress rates for other systems at point $r$ and may take into account several material parameters like the Burgers vector modulus ot the mean free path of dislocation $s$ in the cell (MULLER et al. [12]).

\subsection{Convective hardening}

A heterogeneous incompatible plastic strain field, resulting both from obstacles to dislocation motion and induced microstructure, leads usually to internal stresses whose calculation is classical for static fields (KRÖNER [1]).

In case of plastic flow, the time-derivative $d \sigma / \mathrm{dt}$ of the stress field $\sigma$ incorporates the convective parts caused by plastic gradients of $\beta^{p}$, this accounts for convective motion of the dislocations or gradients of $\beta^{p}$ which are immobile with respect to some parts of the crystal lattice, but mobile relatively to a fixed reference frame in which the usual mechanical variables are described.

When the $\beta^{p}$ field is strongly heterogeneous indeed discontinous, the convective motion of dislocations may contribute substantionally to the internal stresses creating in this way a (non-local) convective kinematic hardening.

Through usual Green functions method, the classical integrals giving the displacement field $u_{n}(r, t)$, and its gradient $u_{n, m}(r, t)$ as a function of the given displacement $u^{0}$ at boundaries and the plastic distortion field $\beta^{p}(r, t)$ are obtained. 
Let the $\beta^{p}$ field present discontinuities on interfaces $\Sigma$ and $v_{n}^{0}$ correspond to a macro-homogeneous loading defined by $v_{n}^{0}(r, t)=G_{n q}(t)_{X_{q}}$ on $S$, the expressions of velocity and velocity gradient follow from GERMAIN [13] :

$$
\begin{aligned}
& v_{n}(r, t)=G_{n q}(t) x_{q}-\int_{V} C_{i j k 1} \mathcal{G}_{j n, i}(R) \frac{\partial \beta_{k l}^{p}}{\partial t}\left(r^{\prime}, t\right) d V^{\prime}-\int_{S} C_{i j k 1} \mathcal{G}_{j n, i}(R) \beta_{k l}^{p}\left(r^{\prime}, t\right) G_{p q}(t) x_{q}^{\prime} n_{p}^{S} d S^{\prime} \\
& +\int_{\Sigma} C_{\mathrm{j} j \mathrm{k} 1} \mathcal{G}_{\mathrm{j}, \mathrm{i}}(\mathrm{R})\left[\beta_{\mathrm{kl}}^{\mathrm{p}}\right] \mathrm{v}_{\mathrm{p}}\left(\mathrm{r}^{\prime}, \mathrm{t}\right) \mathrm{n}_{\mathrm{p}}^{\Sigma} \mathrm{d} \Sigma^{\prime},
\end{aligned}
$$

where $\overrightarrow{\mathrm{n}}^{\mathrm{S}}$ is the unit normal to $S, \overrightarrow{\mathrm{n}}^{\Sigma}$ the unit normal to $\Sigma$, and $\left[\beta_{\mathrm{kl}}^{\mathrm{p}}\right]$ the jump of $\beta^{\mathrm{p}}(\mathrm{r})$ when crossing $\Sigma$, $R=r-r^{\prime}$.

On the other hand, $\mathrm{g}_{\mathrm{nm}}=\mathrm{v}_{\mathrm{n}, \mathrm{m}}$ is expressed through

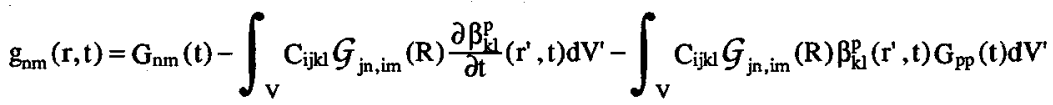

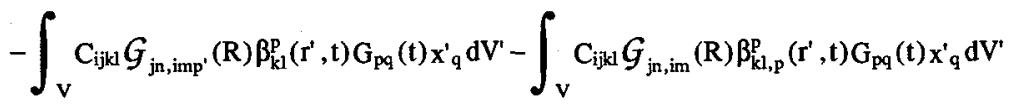

$$
\begin{aligned}
& +\int_{\Sigma} C_{\mathrm{ijk}} \mathcal{G}_{\mathrm{j}, \mathrm{im}}(\mathrm{R})\left[\beta_{\mathrm{kl}}^{\mathrm{p}}\right] \mathrm{v}_{\mathrm{p}}\left(\mathrm{r}^{\prime}, \mathrm{t}\right) \mathrm{n}_{\mathrm{p}}^{\Sigma} \mathrm{d} \Sigma^{\prime}
\end{aligned}
$$

From (16) it is obvious that the velocity gradient depends on plastic strain, which is not the case in the usual descriptions, for the velocity gradient, restricted to the two first terms of equation (16).

\subsection{Application to the dislocation cell structure}

A standard application of integral equation (16) is the problem of a plastic inclusion embedded in a homogeneous elastic matrix. In that case the $\beta^{\mathrm{p}}$ field is piece wise constant in the medium.

Then (16) becomes

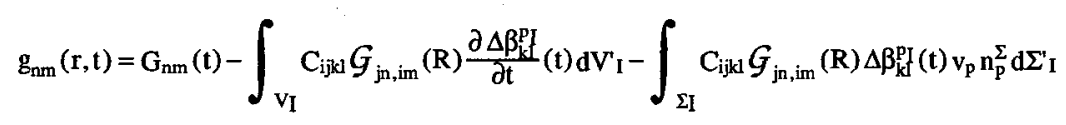

where $\Delta \beta_{\mathrm{kf}}^{\mathrm{p}}$ is the jump between plastic strain tensors in the matrix and in the inclusion.

Moreover, if the inclusion is of an ellipsoidal shape, ESHELBY [14] predicted for the static problem (SABAR et al. [15] for the evolution problem) a uniform gradient $g_{n m}$ in $V_{I}$. In that case, $v_{p}$ on $V_{I}$ can be written in the following form :

$$
\mathrm{v}_{\mathrm{p}}=\mathrm{g}_{\mathrm{pq}}^{\mathrm{I}} \mathrm{x}_{\mathrm{q}}
$$

and (17) becomes, for $r \in V_{I}$,

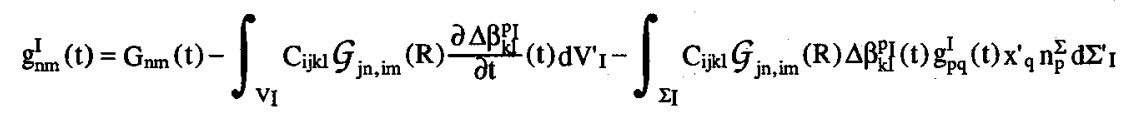

By noting

$$
S_{n m k 1}^{1}=-\int_{V_{I}} C_{i j k 1} \mathcal{G}_{j n, i m}(R) d V^{\prime} \quad \text { and } \quad S_{n m k l p q}^{2}=-\int_{V_{I}} C_{i j k 1} \mathcal{G}_{j n, i m p}(R) x_{q}^{\prime} d V^{\prime}
$$


the gradient $\mathrm{g}^{\mathrm{I}}$ in the inclusion is given by :

$$
\mathrm{g}_{\mathrm{nm}}^{\mathrm{I}}=\mathrm{A}_{\mathrm{nmpq}}\left(\mathrm{G}_{\mathrm{pq}}+\mathrm{S}_{\mathrm{pqk} \mathrm{l}}^{1} \Delta \dot{\boldsymbol{\beta}}_{\mathrm{kl}}^{\mathrm{P}}\right)
$$

where

$$
A_{\text {nmpq }}=\left(\delta_{n q} \delta_{m p}-S_{\text {nmkl }}^{1} \Delta \beta_{k 1}^{p l} \delta_{p q}+S_{\text {nmklpq }}^{2} \Delta \beta_{\mathrm{kl}}^{\text {pl }}\right)^{-1}
$$

The tensor $S^{1}$ has been introduced by Eshelby [14] and the new six-order tensor $S^{2}$ links the velocity gradient to the plastic strain tensor.

On the other hand, by considering a two-phase problem where the soft phase $\mathrm{S}$ (resp. the hard part $\mathrm{H}$ ) is described by the inclusion I (resp.the matrix M), the internal stresses in the dislocation cell structure become from (21)

$$
\begin{aligned}
& \dot{\sigma}^{\mathrm{S}}=\mathrm{C}: A: G-K_{S s}: \dot{\beta}^{\mathrm{pS}}-\mathrm{K}_{\mathrm{SH}}: \dot{\beta}^{\mathrm{pH}_{\mathrm{H}}} \\
& \dot{\sigma}^{\mathrm{H}}=\frac{1}{1-\mathrm{f}} \mathrm{C}:\left(\mathrm{II}_{4}-\mathrm{f} A\right): G-\mathrm{K}_{\mathrm{HS}}: \dot{\beta}^{\mathrm{pS}}-\mathrm{K}_{\mathrm{HH}}: \dot{\beta}^{\mathrm{PH}},
\end{aligned}
$$

where $\mathrm{K}_{\alpha \beta}(\alpha, \beta=S, H)$ are kinematic tensors.

In the same way, one expresses the evolution of the critical shear stresses $i_{c \alpha}^{8}(\alpha=S$ or $H)$ on slip system $g$ as a function of the rates of plastic glides $\dot{\gamma}^{h}$ on slip systems $h$ through the non-local interacting matrices $\mathrm{H}_{\alpha \beta}$ :

$$
\begin{aligned}
& \dot{\tau}_{\mathrm{cS}}^{g}=\mathrm{H}_{S \mathrm{~S}}^{h} \dot{\gamma}_{\mathrm{S}}^{h}+\mathrm{H}_{\mathrm{SH}}^{g h} \dot{\gamma}_{\mathrm{H}}^{h} \\
& \dot{\tau}_{\mathrm{cH}}^{g}=\mathrm{H}_{\mathrm{HS}}^{g h} \dot{\gamma}_{\mathrm{S}}^{h}+\mathrm{H}_{\mathrm{HH}}^{g h} \dot{\gamma}_{\mathrm{H}}^{h}
\end{aligned}
$$

$\mathrm{H}_{\mathrm{HS}}$ and $\mathrm{H}_{\mathrm{HH}}$ are obtained from a generalized dislocation storage-annihilation balance law (MUGHRABI [16]).

$$
\mathrm{H}_{\mathrm{HS}}^{g h}=\frac{\mathbf{b} \mu^{2}}{\mathbf{L}^{h}\left(\tau_{\mathrm{cH}}^{g}-\tau_{\mathrm{cH}}^{8}\right)} \mathbf{a}_{g^{h}} \quad \text { and } \quad \mathrm{H}_{\mathrm{HH}}^{g h}=-\frac{\mathbf{y}_{\mathrm{c}} \rho_{\mathrm{H}}^{h}}{\mathbf{b}\left(\tau_{\mathrm{cH}}^{g}-\tau_{\mathrm{cH}}^{g}\right)} \mathbf{a}_{g h} \text {, }
$$

where $b$ is the Burgers vector modulus, $L$ the mean free path of dislocations in the cell, $\mu$ the shear modulus, $y_{c}$ an annihilation factor, $a_{g h}$ describes the anisotropy of the dislocations and $\rho$ is the dislocation density.

The Schmid conditions applied to this composite allow to obtain the global behavior of the grain

$$
\dot{\Sigma}=L^{\text {eff }}: G \text {, }
$$

where $L^{\text {eff }}$ are the instantaneous elastoplastic moduli of the grain.

This is introduced in a classical local self-consistent scheme in order to get the polycrystalline behavior.

\section{AKNOWLEDGEMENTS}

This paper represents a collective work in which P. LIPINSKI, E. REUBREZ, D. MULLER and X. LEMOINE are strongly implied. 


\section{REFERENCES}

1. KRÖNER, E., Kontinuumstheorie der Versetzungen und Eigenspannungen, Springer , 1958.

2. HILL, R., J. Mech. Phys. Solids 13 (1965) 89.

3. BERVEILLER, M., ZAOUI, A., J. Mech. Phys. Solids 26 (1979) 325, .

4. BERVEILLER, M., ZAOUI, A., J. Engng. Mat. Tech. 106 (1984) 295.

5. LIPINSKI, P., BERVEILLER, M., Int. Journal of Plasticity 5 (1989) 172.

6. KRÖNER, E., J. Engng Mech. Division ASCE 106 (1980) 889.

7. LIPINSKI, P., KRIER, J., BERVEILLER, M., Revue Phys. Appl. 25 (1990) 361.

8. REUBREZ, E., BER VEILLER, M., LIPINSKI, P., Proc. Int. Conf. RISO 92 (1992) 403.

9. SCACCIATELLA, E., Proc. Int. Conf. PLASTICITY 91, GRENOBLE (1991) 659.

10. SCHMITT, C., LIPINSKI, P., BERVEILLER, M., proposed at Int. J. Plasticity, 1993.

11. BERVEILLER, M., MULLER, D., KRATOCHVIL, J., to appear in Int. J. of Plast., 1993.

12 MULLER, D., LEMOINE, X., BERVEILLER, M., KRATOCHVIL, J., Int. Symp. Disl.93, Aussois, France, march 1993.

13. GERMAIN, P., Mécanique des Milieux continus, Ed Masson et Cie Paris, 1973.

14. ESHELBY, J.D., Prog. in Sol. Mech. North Holland, Amsterdam t.2 (1961) 89.

15. SABAR, H., BUISSON, M., BERVEILLER, M., Int. J. Plasticity 7 (1991) 759.

16. MUGHRABI, H., Revue Phys. Appl. 23 (1988) 367. 